\title{
Effektive und sichere Varizenbehandlung mit Schaum
}

- Seit kurzem kann Aethoxysklerol zur Schaumsklerosierung verwendet werden. Damit ist Schaum als zusätzliche Anwendungsart von Aethoxysklerol offiziell genehmigt und anerkannt. Bereits vor der offiziellen Zulassung galt die Schaumsklerosierung von Varizen, laut Leitlinie der Deutschen Gesellschaft für Phlebologie, als anerkannte Standardtherapie. Mit der Zulassung wurde diese Empfehlung nun bestätigt. „Zur Behandlung mittlerer und großer Varizen stellt die Schaumsklerosierung eine sehr effektive und sichere Methode dar, die aufgrund der ausgezeichneten Datenlage nun auch offiziell anerkannt wurde", fasst Prof. Eberhard Rabe, Bonn, die Ergebnisse zusammen.

Die erste vergleichende, randomisierte kontrollierte klinische Multizenterstudie vergleicht die Sicherheit und Wirksamkeit von standardisiertem Polidocanol-Schaum mit flüssigem Polidocanol bei der Sklerotherapie der Vena saphena magna [Eur J Vasc Endovasc Surg 2008; 35, 238-45]. Die Studie wurde in elf deutschen Venenzentren an 106 Patienten zwischen 18 und 70 Jahren durchgeführt. Einschlusskriterium war eine Varikose aufgrund einer insuffizienten Vena saphena magna. Klinisch lagen Venenprobleme der Kategorien C2 bis $C_{5}$ nach CEAP-Klassifikation vor. Die Patienten wurden entweder mit standardisiertem Polidocanol-Schaum (3\%) oder mit flüssigem Polidocanol (3\%) behandelt. Der standardi- sierte Schaum wurde mit dem Spritzensys-

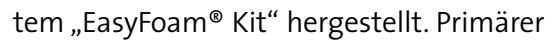
Endpunkt zur Bestimmung der Wirksamkeit war die Ausschaltung des Reflux definiert als $<0,5$ Sekunden.

Die Studie zeigte, dass ein signifikant höherer Anteil an Patienten erfolgreich mit Schaum behandelt wurde: Im Vergleich zu $27 \%$ bei der Flüssigsklerosierung gab es in der „Schaum-Gruppe“ 69\% Responder ( $p<$ o,0001). Auch die Patientenzufriedenheit war in der Gruppe der mit Schaum Behandelten signifikant höher $(p=0,0008)$. Di- ese Ergebnisse spiegelten sich auch in der Beurteilung durch den behandelnden Arzt wider. Die Anzahl an Therapiesitzungen war mit Schaum signifikant niedriger als mit Flüssigkeit. In Bezug auf Nebenwirkungen wurde kein signifikanter Unterschied zwischen den beiden Behandlungsgruppen beobachtet. Für die Behandlung von Besenreisern und retikulären Varizen hingegen gilt die Flüssigsklerosierung weiterhin als Methode der ersten Wahl.

red

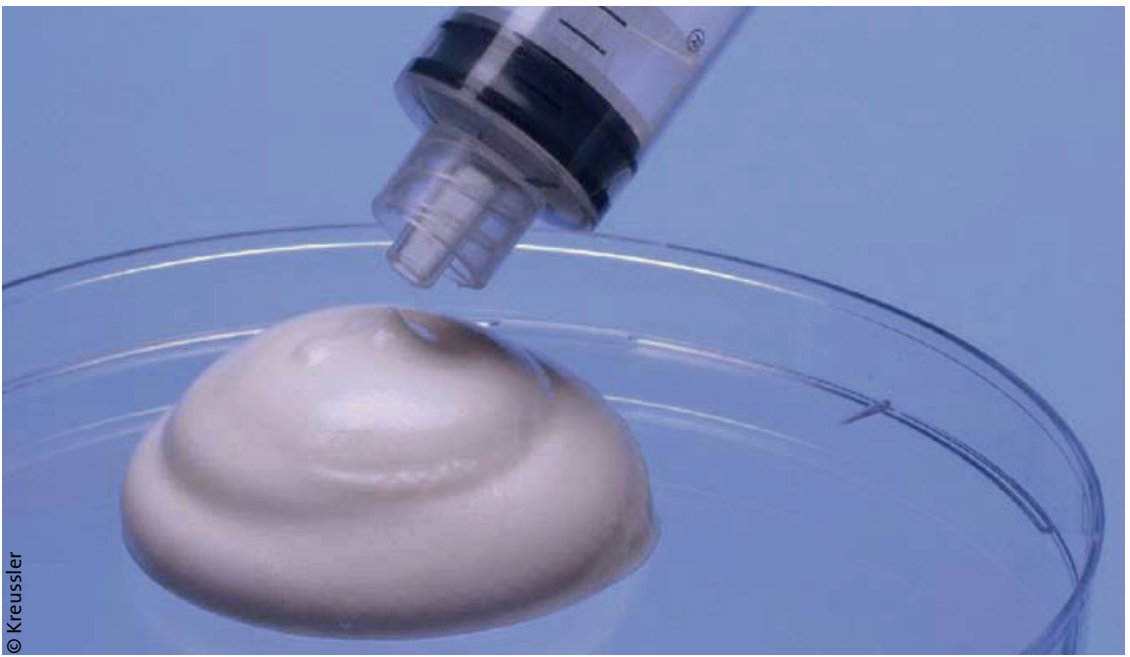

Bei der Schaumsklerosierung wird aus dem flüssigen Sklerosierungsmittel in den Konzentrationen 1, 2 und 3\% Polidocanol mittels einer speziellen Technik ein feinblasiger, standardisierter Sklerosierungsschaum hergestellt.

\section{Neue systemische Therapieoption bei Rosacea}

- Rosacea erfordert eine phasen- und stadiengerechte Behandlung. Für die papulopustulöse Rosacea bei Erwachsenen hat sich in jüngster Zeit ein neuer Therapieansatz mit subantimikrobiell dosiertem Doxycyclin etabliert. Dieser beruht ausschließlich auf anti-inflammatorischen Wirkprinzipien und ermöglicht eine sichere und wirksame Kontrolle der Erkrankung. In der neuen, teilretardierten Formulierung von Doxycyclin (Oraycea ${ }^{\circledR}$ ) werden $30 \mathrm{mg}$ des Wirkstoffs schnell und $10 \mathrm{mg}$ langsam freigesetzt. Dadurch wird eine Gleichge- wichtskonzentration von Doxycyclin erzielt, die im entzündungshemmenden, aber nicht antimikrobiell wirksamen Bereich bleibt. Daraus resultiert nach Einmalgabe beziehungsweise unter Steady-state-Bedingungen (nach sieben Tagen) ein über 24 Stunden konstanter Wirkstoffspiegel im Blutplasma. Bei einmal täglicher Anwendung gewährleistet diese subantimikrobielle Dosierung eine kontinuierliche anti-inflammatorische Wirksamkeit. Eine Vergleichsstudie mit 100 mg Doxycyclin und subantimikrobiell dosiertem Doxy- cyclin bei Rosacea belegt: Die Wirksamkeit von Doxycyclin in einer antientzündlichen, nicht antibiotischen Dosierung ist genauso stark wie die von $100 \mathrm{mg}$ Doxycyclin, jedoch bei signifikant besserem Nebenwirkungsprofil (weniger antibiotikatypische Nebenwirkungen wie etwa vaginale Candidosen, gastrointestinale Beschwerden und Photosensibilisierungen) und ohne bakterielle Resistenzen zu induzieren. red

Nach Informationen von Galderma 